\title{
Rod Photoreceptor Neurite Sprouting in Retinitis Pigmentosa
}

\author{
Zong-Yi Li,' Ivar J. Kljavin, ${ }^{2}$ and Ann H. Milam' \\ ${ }^{1}$ Department of Ophthalmology, University of Washington, Seattle, Washington 98195 and ${ }^{2}$ Genentech, South San \\ Francisco, California 94080
}

In animal models for retinitis pigmentosa (RP), rod photoreceptors show abnormal distribution of rhodopsin prior to undergoing cell death. To elucidate the steps in degeneration of human photoreceptors, immunocytochemistry was performed on donor retinas from 15 RP patlents and flve normal subjects. Rhodopsin immunolabeling in the normal retinas was restricted to the rod outer segments. In the RP retinas, rhodopsin was present in shortened rod outer segments and in the surface membranes of the rod inner segments and somata. In regions of photoreceptor death, the surviving rods had sprouted rhodopsin-positive neurites that were closely associated with gliotic Müller cell processes and extended to the inner limiting membrane. Rods and cones in the RP maculas did not form neurites, but the axons of peripheral cones were abnormally elongated and branched. Double immunofluorescence labeling showed that the rod neurites bypassed the horizontal and rod bipolar cells that are normally postsynaptic to rod axons. To our knowledge, this is the first report of rod neurite sprouting in vivo. We were unable to find neurites on degenerate rods in old rds mice, an animal model for RP. The rod neurites in the human RP retinas resemble the long, branched processes formed by rods cultured on Müller cells or purified $\mathrm{N}$-CAM. Neurite growth by surviving rods in the RP retinas may be a response to neurotrophic factor upregulation, loss of inhibitory factors, or changes in molecules associated with reactive Müller cells. Such changes in the retinal microenvironment may impede functional integration of transplanted photoreceptors. The contributions of the rhodopsinpositive rod neurites and abnormal cone axons to the functional abnormalities observed in RP are unknown.

[Key words: retina, rod, cone, neurites, Müller glia, retinitis pigmentosa]

Received Feb. 20, 1995; revised Apr. 12, 1995; accepted Apr. 13, 1995.

This work was supported by the Foundation Fighting Blindness, Baltimore, MD, NIH Grants EY01311 and EY01730, and by an award from Research to Prevent Blindness, Inc. (RPB), New York, NY. A.H.M. is a Senior Scientific Investigator of RPB. Legal requirements for use of human donor postmortem tissues were met (University of Washington Human Subjects Approval \#25034-E, dated 02/01/95). Human donor eyes were provided by the Foundation Fighting Blindness, and the University of Washington Lions' Eye Bank, supported by the Northern Idaho Lions' Sight Conservation Foundation, Seattle, WA. We thank Ms. J. Chang, Mr. D. Possin, and Ms. I. Klock for technical assistance; Mr. C. Stephens and Mr. R. Jones for photographic help; Dr. J. McGinnis for the $r d s$ mouse eyes; the scientists listed in the Materials and Methods section who provided antibodics; and Drs. J. Saari and A. Latics for critical review of the manuscript.

Correspondence should be addressed to Zong-Yi Li, MD, Department of Ophthalmology RJ-10, University of Washington, Seattle, WA 98195.

Copyright 101995 Society for Neuroscience $0270-6474 / 95 / 155429-10 \$ 05.00 / 0$
Retinitis pigmentosa (RP) is a group of inherited diseases that cause degeneration of rod and cone photoreceptors, reactive changes in the retinal pigment epithelium and Müller glia, and atrophy of blood vessels and neurons in the inner retina (Stone et al., 1992; Li et al., 1995). RP is associated with mutations in several photoreceptor-specific genes, including rhodopsin (Dryja, 1992; Humphries et al., 1993), but many gaps remain in our understanding of the mechanisms of photoreceptor dysfunction and death. Recent studies of mice carrying mutant rhodopsin transgenes demonstrated that their rods show abnormal localization of rhodopsin, transducin, and phosphodiesterase prior to undergoing cell death (Roof et al., 1994; Sung et al., 1994). To elucidate the process of photoreceptor cell death in humans, we have screened postmortem RP retinas by immunocytochemistry, using antibody markers that are specific for rods and cones, inner retinal neurons, and Müller cells. We present evidence that rod photoreceptors in these retinas form long, rhodopsin-positive neurites that extend for considerable distances into the inner retina. Although rods are known to form long neurites in culture (Araki et al., 1987; Kljavin and Reh, 1991; Gaur et al., 1992; Mandell et al., 1993; Hicks et al., 1994; Kljavin et al., 1994), to our knowledge this is the first report of rod neurite sprouting in vivo. While rod neurite sprouting is common in the human $\mathrm{RP}$ retinas, this phenomenon is not seen in several animal models of RP, including older $r d s$ mice examined in the present study. We demonstrate that the rod neurites extend past the neurons that normally receive rod synaptic input and are closely associated with gliotic Müller cells in the RP retinas. Finally, we discuss the possibilities that the rod neurites are formed in response to neurotrophic factor upregulation, lack of inhibitory factors in the diseased retinas, or alterations in molecules associated with the reactive Müller glia.

\section{Materials and Methods}

Tissue preparation. Postmortem eyes were obtained through the donor program of the Foundation Fighting Blindness, Baltimore, MD, from $15 \mathrm{RP}$ paticnts who ranged in age from 24 to 89 years (Table 1). Eyes from five normal subjects, aged 51-91 years, were obtained through the Foundation and the University of Washington Lions' Eye Bank. The eyes had been fixed for 4 weeks to 4 years in $0.13 \mathrm{M}$ phosphate-buffered $4 \%$ paraformaldehyde and $0.5 \%$ glutaraldehyde, or in phosphate-buffered $4 \%$ paraformaldehyde alone.

Eyes from 10- and 16-month-old retinal degeneration slow $(r d s)$ mice were obtained from Dr. James McGinnis, University of California Los Angeles. The eyes had been fixed in Perfix (Fisher, Santa Clara, CA) for $4 \mathrm{hr}$ at $4^{\circ} \mathrm{C}$ and stored in $70 \%$ ethanol. The retinas were processed through a descending ethanol series into phosphate buffer and treated as below for indirect immunofluorescence.

Electron microscopy. Tissues were postfixed in 1\% phosphate-buffered osmium tetroxide and embedded in Medcast (Ted Pella, Inc., Redding, CA). Ultrathin sections were stained with uranyl acetate and lead citrate. 
Table 1. Characteristics of donor retinas used in study

\begin{tabular}{llrl}
$\begin{array}{l}\text { Reference } \\
\text { no. }\end{array}$ & Age/gender & $\begin{array}{l}\text { PM time } \\
\text { (hr) }\end{array}$ & Diagnosis \\
\hline FFB-310 & $24 \mathrm{M}$ & 11.5 & Usher syndrome \\
FFB-342 & $29 \mathrm{M}$ & 16.0 & Simplex RP \\
FFB-114 & $39 \mathrm{M}$ & 5.5 & XL RP \\
FFB-311 & $44 \mathrm{M}$ & 12.0 & Simplex RP \\
FFB-215 & $46 \mathrm{M}$ & 1.0 & XL RP \\
FFB-424 & $50 \mathrm{~F}$ & 6.5 & AD RP \\
FFB-231 & $56 \mathrm{M}$ & 3.3 & Simplex RP \\
FFB-335 & $58 \mathrm{~F}$ & 5.5 & AD RP \\
FFB-303 & $68 \mathrm{M}$ & 2.2 & AD RP \\
FFB-316 & $68 \mathrm{M}$ & 8.5 & AD RP \\
FFB-271 & $72 \mathrm{~F}$ & 4.0 & XL RP carrier \\
FFB-340 & $73 \mathrm{~F}$ & 9.0 & Simplex RP \\
FFB-184 & $76 \mathrm{M}$ & 3.1 & Multiplex RP \\
FFB-356 & $80 \mathrm{M}$ & 5.0 & AD RP \\
FFB-371 & $87 \mathrm{M}$ & 5.0 & Simplex RP \\
UW-403-93 & $51 \mathrm{~F}$ & 3.0 & Nornlal \\
UW-805-92 & $53 \mathrm{~F}$ & 3.0 & Normal \\
FFB-343 & $66 \mathrm{M}$ & 2.5 & Normal \\
UW-780-91 & $85 \mathrm{~F}$ & 3.0 & Normal \\
FFB-363 & $91 \mathrm{~F}$ & 3.3 & Normal \\
\hline
\end{tabular}

FFB, Foundation Fighting Blindness; PM, postmortem; $\mathrm{XL}, \mathrm{X}$ linked; AD, autosomal dominant; UW, University of Washington.

a Rhodopsin glutamine-64-ter mutation.

${ }^{b}$ Rhodopsin threonine-17-methionine mutation ( $\mathrm{Li}$ et al., 1994).

Immunocytochemistry. Tissue samples were held in $30 \%$ phosphatebuffered sucrose overnight, cryosectioned at $12 \mu \mathrm{m}$, and processed for indirect immunofluorescence according to published methods (Milam and Jacobson, 1990). Some aldehyde-fixed tissues were treated with sodium borohydride, embedded in LR-White resin (Ted Pella, Inc.), and processed by the immunogold technique with silver enhancement for light microscopy ( $1 \mu \mathrm{m}$ sections) and electron microscopy (90 nm sections) (Milam and Jacobson, 1990).

Antibodies. The following antibodies against rhodopsin were used: monoclonal antibodies 1D4 (against the C-terminus, 1:20) and 4D2 (against the N-terminus, 1:20) from Dr. R. Molday, University of British Columbia, Vancouver, Canada; polyclonal anti-rhodopsin $(1: 200)$ from Dr. T. Shuster, California State University at Long Beach, CA; polyclonal anti-rhodopsin (1:5000) from Dr. E. Kean, Case Western Reserve University, Cleveland, $\mathrm{OH}$; and monoclonal antibody P-Rho (1:5) against phosphorylated rhodopsin from Dr. P. Hargrave, University of Florida, Gainesville, FL. Antibodies against other rod proteins were polyclonal anti-arrestin (1:50) from Dr. H. Shichi, Wayne State University, Detroit, MI; monoclonal anti-cGMP channel protein (PMc 1D1, undiluted) and monoclonal anti-rds/peripherin (3B6 and $5 \mathrm{H} 2$, undiluted) from Dr. R. S. Molday; polyclonal anti-recoverin (1:100) from Dr. A. Dizhoor, University of Washington, Seattle, WA; monoclonal anti-ROM-1 (1:1000) from
Dr. R. McInnes, Hospital for Sick Children, Toronto, Canada; polyclonal anti-rhodopsin kinase $(1: 100)$ from Dr. K. Palczewski, University of Washington; monoclonal anti-transducin $\alpha$-rod (undiluted) from Dr. J. Saari, University of Washington; and polyclonal anti-transducin $\alpha$-cone (1:10), polyclonal anti-red/green cone opsin (1:100), and polyclonal antiblue cone opsin (1:10) from Drs. C. and K. Lerea, New York Medical College, Valhalla, NY. Antibodies against synaptic vesicle proteins were monoclonal anti-synaptophysin (1:200; Sigma, St. Louis, MO) and monoclonal anti-SV2 protein (1:400) from Drs. K. Buckley and R. B. Kelly, University of California, San Francisco, CA. Antibody markers for inner retinal neurons were polyclonal anti-L7 protein (1:200) from Dr. J. Morgan, Hoffmann LaRoche, Inc., Nutley, NJ; and monoclonal anti-calbindin (1:200; Sigma). The marker for reactive Müller cells was polyclonal antiglial fibrillary acidic protein (GFAP; 1:200; Dako Corporation, Carpinteria, CA).

\section{Results}

\section{Light microscopic immunocytochemistry of human retinas}

Normal retinas processed for immunofluorescence showed heavy labeling of the rod outer segments with each of the antibodies against rhodopsin (Fig. 1A). Rods in the normal retinas also showed outer segment labeling with antibodies against the cGMP channel, rds/peripherin, rhodopsin kinase, ROM-1, and transducin $\alpha$-rod. The rod outer segments, inner segments, and somata were reactive with anti-arrestin and -recoverin. Cone outer segments were labeled with the antibody against $r d s$ /peripherin and the red/green and blue cone opsins; cone outer segments, inner segments, and cell bodies were reactive with antirecoverin and -transducin $\alpha$-cone. The outer and inner plexiform layers were labeled with anti-SV-2 (Fig. $2 A$ ), and anti-synaptophysin labeled the cone inner segments and somata, in addition to the two plexiform layers. Anti-calbindin labeled cones, horizontal cells, and some neurons in the inner nuclear layer, and anti-L7 specifically labeled the rod bipolar cells.

In all RP retinas, the rod outer segments were markedly shortened and were reactive with the antibodies against rhodopsin (Fig. 1B), phosphorylated rhodopsin, the cGMP channel, rds/ peripherin (Fig. 1C), and ROM-1. Rod inner segments and somata were reduced in number and were labeled with the antibodies against rhodopsin, phosphorylated rhodopsin, arrestin, recoverin (Fig. $1 D$ ), rhodopsin kinase, and transducin $\alpha$-rod. Cone outer segments were also shortened and were labeled with the antibodics against the red/grecn and blue cone opsins, $r d s /$ peripherin, and transducin $\alpha$-cone (Fig. 2C). The cone inner segments and cell bodies were reduced in number and were labeled with the antibodies against calbindin, recoverin, transducin $\alpha$-cone (Fig. 2C), and synaptophysin (Fig. 2D).

A striking abnormality was evident in all of the RP retinas. In the peripheral regions where the photoreceptors were reduced to one to three rows of cells, the rods gave rise to long neurites that

Figure 1. Normal and RP human retinas labeled by immunofluorescence. $A$, In the normal retina, anti-rhodopsin labels the rod outer segments (ROS). Arrow, inner limiting membrane. B, In an RP retina, rhodopsin is localized to the short rod outer segments (arrowheads), inner segments, and somata $(S)$. Rhodopsin is also localized in the beaded rod neurites (open arrow) that extend from the bases of the rods to the inner limiting membrane (closed arrow). $C$, In an area of an RP retina containing rod neurites, anti-rds/peripherin labels the short photoreceptor outer segments (arrowheads) but not the rod neurites. $D$, In an RP retina, anti-recoverin labels the photoreceptor outer (arrowheads) and inner segments and somata, as well as the rod neurites (arrows). E, The macula of an RP retina immunolabeled for the demonstration of rhodopsin. The remaining rods show surface membrane labeling of their somata (arrowheads) but no rod outer segments or neurites are present. $F$, The macula of an RP retina immunolabeled for the demonstration of transducin $\alpha$-cone. The cone somata (arrowheads) are labeled but there is no evidence of cone neurite sprouting. $G$, The midperipheral retina from a man with X-linked RP (FFB-215) immunolabeled with anti-rhodopsin. Considerable loss of rods has occurred and the remaining rods have sprouted neurites. The retina is partially detached from the retinal pigment epithelium $(*) . H$, The far peripheral region of the retina shown in $G$, also immunolabeled with anti-rhodopsin. More rods are retained in this part of the retina and rod neurite sprouting is less extensive than in $G$. The rods have short outer segments (arrows). $I$ and $J$, Double immunolabeling to demonstrate GFAP ( $I$, FITC labeling) and rhodopsin ( $J$, rhodamine labeling) in the same micrographic field. $I$, The Müller cells are hypertrophic and their processes are GFAP positive. $J$, The rhodopsin-positive rod neurites course along the Müller cell processes. Magnification, $230 \times$. 

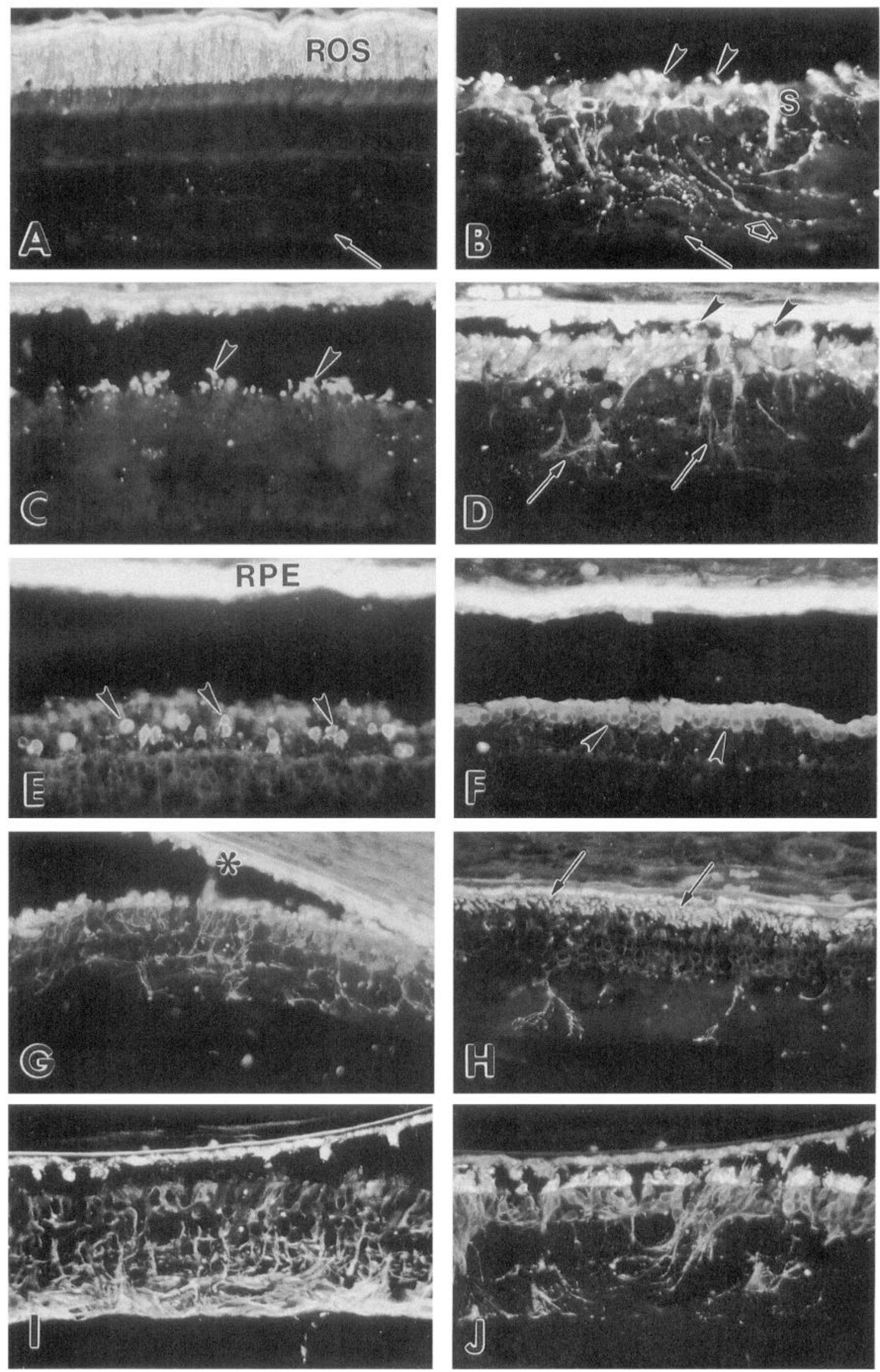

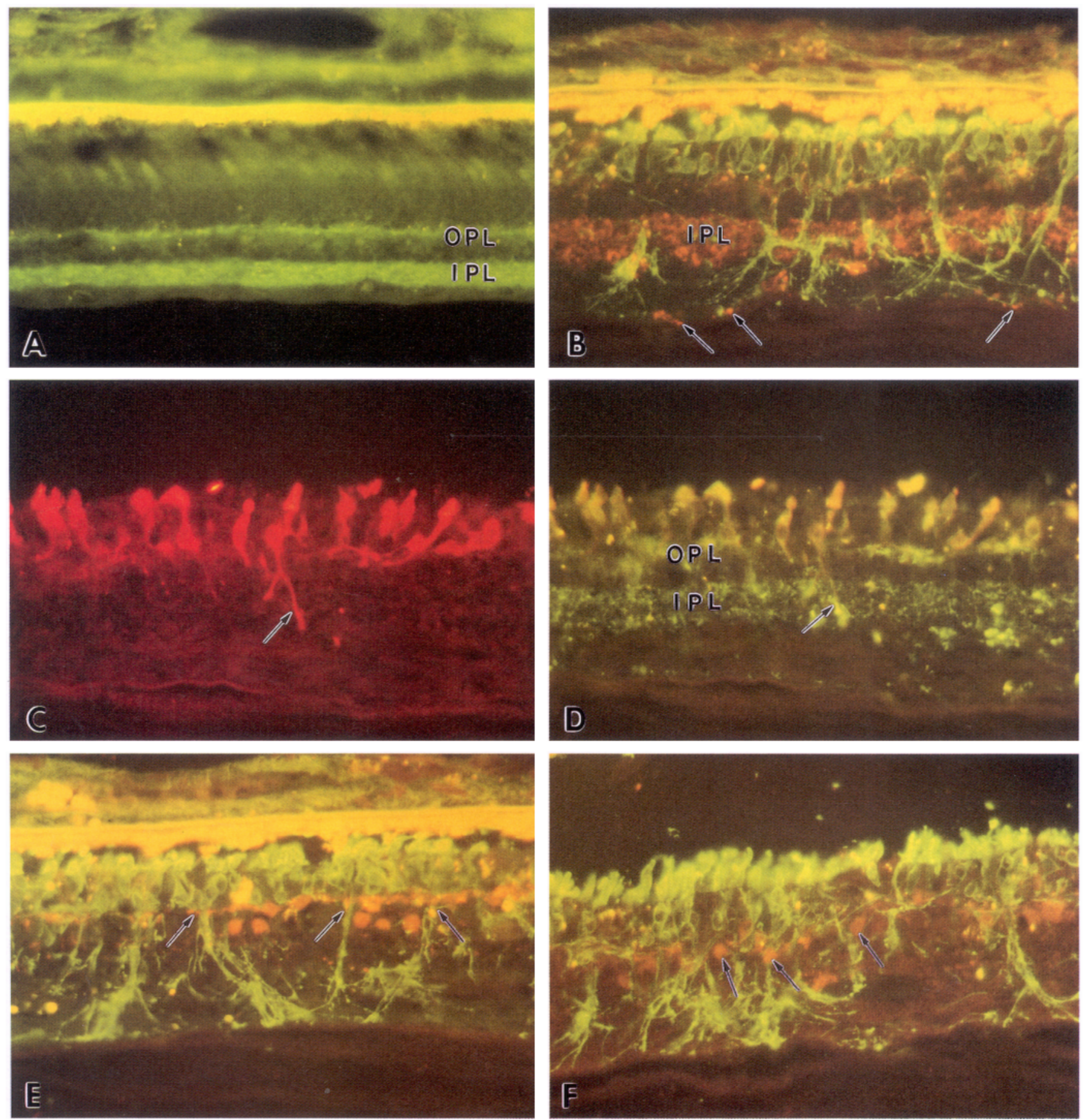

Figure 2. Normal and RP human retinas after immunofluorescence labeling. A, Distribution of SV2 synaptic vesicle protein in the inner (IPL) and outer $(O P L)$ plexiform layers of a normal retina. The yellow band corresponds to autofluorescent lipofuscin in the retinal pigment epithelium. $B$, Double labeling for SV2 (rhodamine, red) and rhodopsin (FITC, green) in an RP retina. The anti-SV2 labels the outer and inner (IPL) plexiform layers, plus bulb-shaped varicosities (arrows) on the rhodopsin-positive rod neurites. $C$, Cones in the peripheral region of an RP retina labeled for the demonstration of transducin $\alpha$-cone, which fills the cone cytoplasm. Some cone axons (arrow) are abnormally long and branched, extending into the inner plexiform layer (see $D$ ). $D$, Section in $C$ double labeled for the demonstration of transducin $\alpha$-cone (rhodamine) and synaptophysin (FITC) in the inner (IPL) and outer (OPL) plexiform layers. The cones are labeled with both antibodies, and the cone axon (arrow) extends into the inner plexiform layer. $E$, Double labeling for calbindin (rhodamine) and rhodopsin (FITC) in an RP retina. The rhodopsin-positive rod neurites pass through the band of calbindin-positive horizontal cell processes (arrows). Five amacrine cells are also labeled with anti-calbindin. $F$, Double labeling of L-7 (rhodamine) and rhodopsin (FITC) in the peripheral region of an RP retina. The rhodopsin-positive rod neurites course between and past the labeled rod bipolar cells (arrows) and reach the inner limiting membrane. Magnification, $280 \times$. 
were labeled with each of the antibodies against rhodopsin and phosphorylated rhodopsin (Fig. 1B). The neurites often had beaded varicosities and clusters of bulb-shaped terminals that were labeled with antibodies against synaptophysin and SV-2 (Fig. 2B). Some of the labeled terminals were found in the innermost part of the retina, between the nerve fiber layer and the inner limiting membrane (Fig. 2B). The rod neurites were also labeled with antibodies against the rod cytoplasmic proteins arrestin, recoverin (Fig. 1D), rhodopsin kinase, and transducin $\alpha$-rod. The short rod outer segments were well labeled with antibodies against $r d s$ /peripherin, ROM-1, and the cGMP channel, but the rod ncurites werc not labeled with these antibodies (Fig. 1C).

The maculas of the RP retinas contained scattered rod somata that lacked outer segments but were labeled with antibodies against rhodopsin (Fig. $1 E$ ) and the rod cytoplasmic proteins. These rods did not form prominent neurites as found in the peripheral regions of the same retinas. Cones in each RP macula were reduced to a monolayer of somala with absent or very short outer segments that were reactive for the cone opsins, recoverin, and transducin- $\alpha$ cone. The cone somata and axons were well labeled with the antibodies against recoverin and transducin $\alpha$-cone (Fig. $1 F$ ), but there was no indication of cone neurite formation in the RP maculas.

Cones in the peripheral parts of the RP retinas showed the same patterns of immunolabeling as found in the maculas. Labeling with the antibody against transducin- $\alpha$ cone revealed that many of the cone axons were abnormally branched and elongated (Fig. $2 C$ ). Double labeling with the antibodies against transducin- $\alpha$ cone and synaptophysin revealed that some cone axons passed through the inner nuclear layer and terminated in the inner plexiform layer (Fig. 2D).

Rod neurite sprouting was present in the retinas of all of the RP patients, including those with known rhodopsin mutations (Table 1) and those with X-linked RP, who presumably had normal rhodopsin because the gene for this protein is located on chromosome 3 (Dryja, 1992). Within a given retina, rod neurite sprouting was most pronounced in regions that had undergone significant photoreceptor death. For example, within the retina of the 46-year-old man with X-linked RP (FFB-215, Table 1), neurite sprouting was extensive in the midperipheral retina (Fig. $1 G$ ), where significant rod cell death had already occurred, while in the far peripheral retina (Fig. $1 H$ ) most rods were still viable and only a few had formed neurites.

Because ganglion cells, the third-order retinal neurons, can be lost in RP (Stone et al., 1992), it seemed possible that the prominent rod neurites had formed in response to transneuronal degeneration of second-order postsynaptic neurons, for example, the horizontal and rod bipolar cells. To test this, double labeling was performed with anti-rhodopsin and specific markers for the horizontal cells (anti-calbindin) and the rod bipolar cells (antiL7 protein). Anti-calbindin labeled a continuous band of horizontal cell processes in the outer plexiform layer in the normal retinas. In the RP retinas, a similar calbindin-labeled band was present and a few of the labeled horizontal cell processes gave rise to abnormal apical sprouts. The rhodopsin-positive rod neurites did not terminate on the horizontal cell processes but passed directly through the labeled band into the inner retina (Fig. 2E). A single row of L7-labeled rod bipolar cells was present in the peripheral regions of both the normal and RP retinas (Fig. $2 F$ ), indicating that this cell type had not undergone significant transneuronal degeneration in response to death of rods. The rhodopsin-positive neurites coursed between and past the labeled rod bipolar somata and terminated against the inner limiting membrane (Fig. $2 F$ ).

GFAP immunolabeling in normal retinas is limited to the astrocytes near the inner limiting membrane (Eisenfeld et al., 1984). As shown previously in other RP retinas (Milam and Jacobson, 1990; Li et al., 1994, 1995), Müller cells undergo reactive gliosis following death of photoreceptors. The Müller processes in the maculas and peripheral regions of the RP retinas were hypertrophic and reactive throughout with anti-GFAP (Fig. $1 I$ ), and some Müller nuclei were enlarged and had migrated from the inner nuclear layer into the photoreceptor layer (not shown). Double labeling of the same micrographic field revealed that the rhodopsin-positive neurites coursed along the GFAPpositive radial Müller processes (Fig. $1 \mathrm{~J}$ ), but there was no indication by light or electron microscopy of Müller cell labeling with anti-rhodopsin.

\section{Electron microscopy of human retinas}

The fine structure of the rods and cones in the normal retinas was as described previously for human photoreceptors (Hogan et al., 1971). The rod and cone axons terminated in the outer plexiform layer as ribbon synapses with numerous small, clear synaptic vesicles $\sim 50 \mathrm{~nm}$ in diameter. Immunogold labeling of the normal rods with anti-rhodopsin was limited to their outer segments (Fig. 3A). Rods in the peripheral regions of the RP retinas had very short outer segments that were well labeled with anti-rhodopsin (Fig. 3B). In addition, rhodopsin labeling was prominent on the surface membranes of the rod inner segments (Fig. 3B), somata, and synapses (Fig. 3C), and in the Golgi regions of the inner segments. No rhodopsin immunoreactivity was found in Müller glia or any other retinal cells. The rod neurites were recognizable in the inner retina by virtue of their surface labeling with anti-rhodopsin (Fig. 4A). By conventional $\mathrm{EM}$, the rod neurites contained numerous longitudinally oriented microtubules, and expanded regions of the neurites corresponding to the beaded varicosities observed by light microscopy were filled with numerous small vesicles that were $\sim 50 \mathrm{~nm}$ in diameter, as well as prominent, dense multivesicular bodies that measured up to $\sim 400 \mathrm{~nm}$ in diameter (Fig. $4 B$ ). The regions of the neurites that contained the small vesicles were immunolabeled with antibodies against synaptophysin and SV-2 synaptic vesicle protein (not shown). No synaptic ribbons were identified in the rod ncurites. The neurites were closely apposed to enlarged Müller cell processes filled with intermediate filaments (Fig. $4 C$ ) that were immunopositive for GFAP (not shown). The rod neurites terminated next to the inner limiting membrane basal lamina and did not extend past this layer into the vitreous humor (Fig. 4A,C).

\section{Light microscopic immunocytochemistry of $\mathrm{rds}$ mouse retinas}

There were no published reports of rod neurite sprouting in the retinas of the animal models for RP, although rhodopsin had been localized in these retinas by immunocytochemistry (Jansen et al., 1987; Nir et al., 1989; Cantera et al., 1990; Nir et al., 1990; Roof et al., 1994; Sung et al., 1994). It seemed that rod degeneration might occur too rapidly in most of the animal models for detection of rod neurites, so we examined the retinas of $10-$ and 16-month-old $r d s$ mice, which have a very slow course of rod degeneration. Immunolabeling with anti-rhodopsin (monoclonal antibodies 4D2 and 1D4) and -recoverin revealed that an incomplete monolayer of rod somata persisted at 10 months but that all rods had degenerated by 16 months. The 


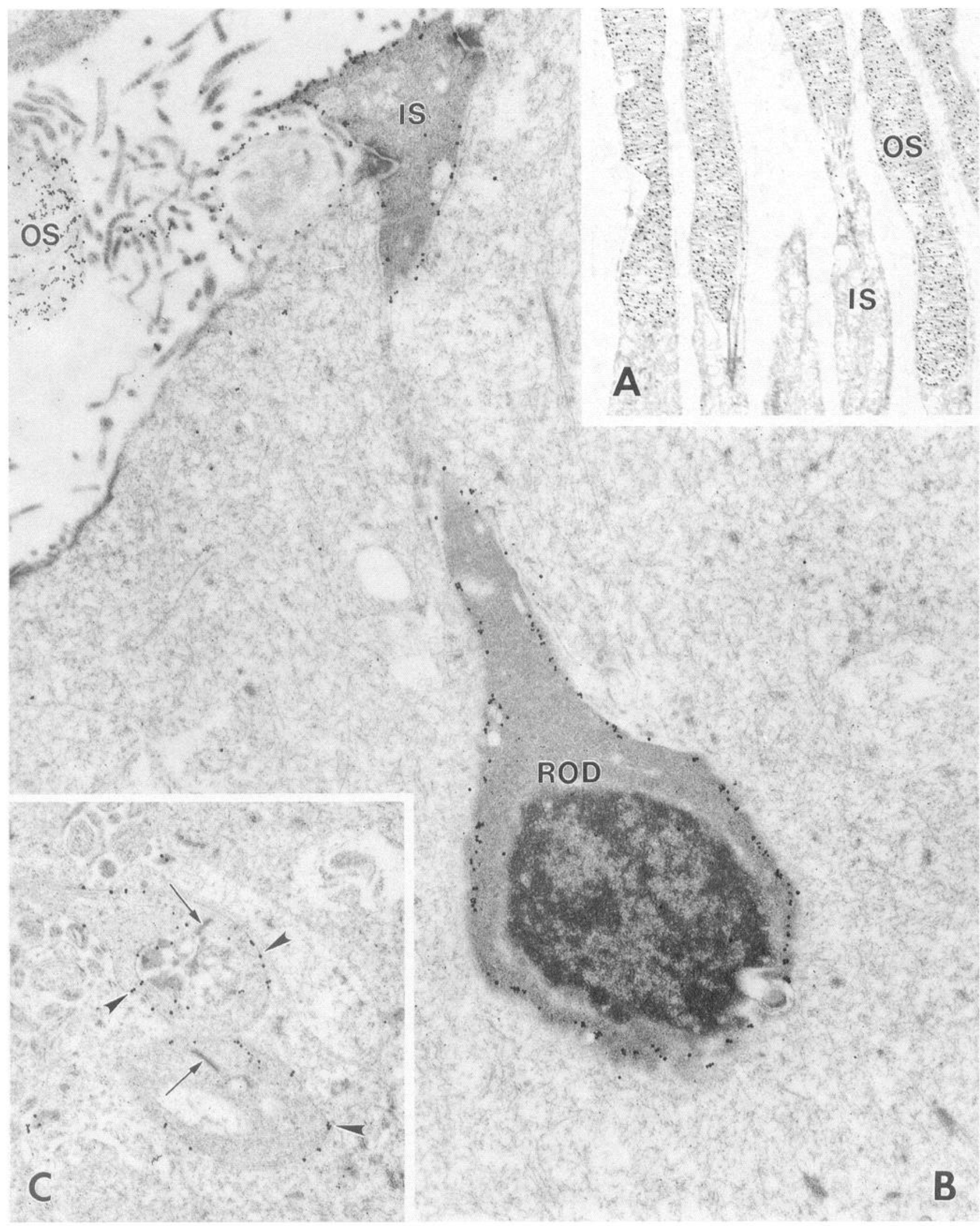

Figure 3. Electron micrographs of normal and RP retinas labeled with anti-rhodopsin. $A$, Normal retina shows labeling with anti-rhodopsin of the rod outer segments $(O S)$. The inner segments $(I S)$ are free of label. $B$, The peripheral region of an RP retina shows labeling with anti-rhodopsin of the short outer segment $(O S)$ and the surface membrane of the inner segment $(I S)$ and rod soma $(R O D)$ ). $C$, The peripheral region of an RP retina shows labeling with anti-rhodopsin of the surface membranes of the rod synapses (arrowheads). Arrows, synaptic ribbons. Magnification: $A, 7,380 \times$; $B, 16,530 \times ; C, 13,350 \times$. 

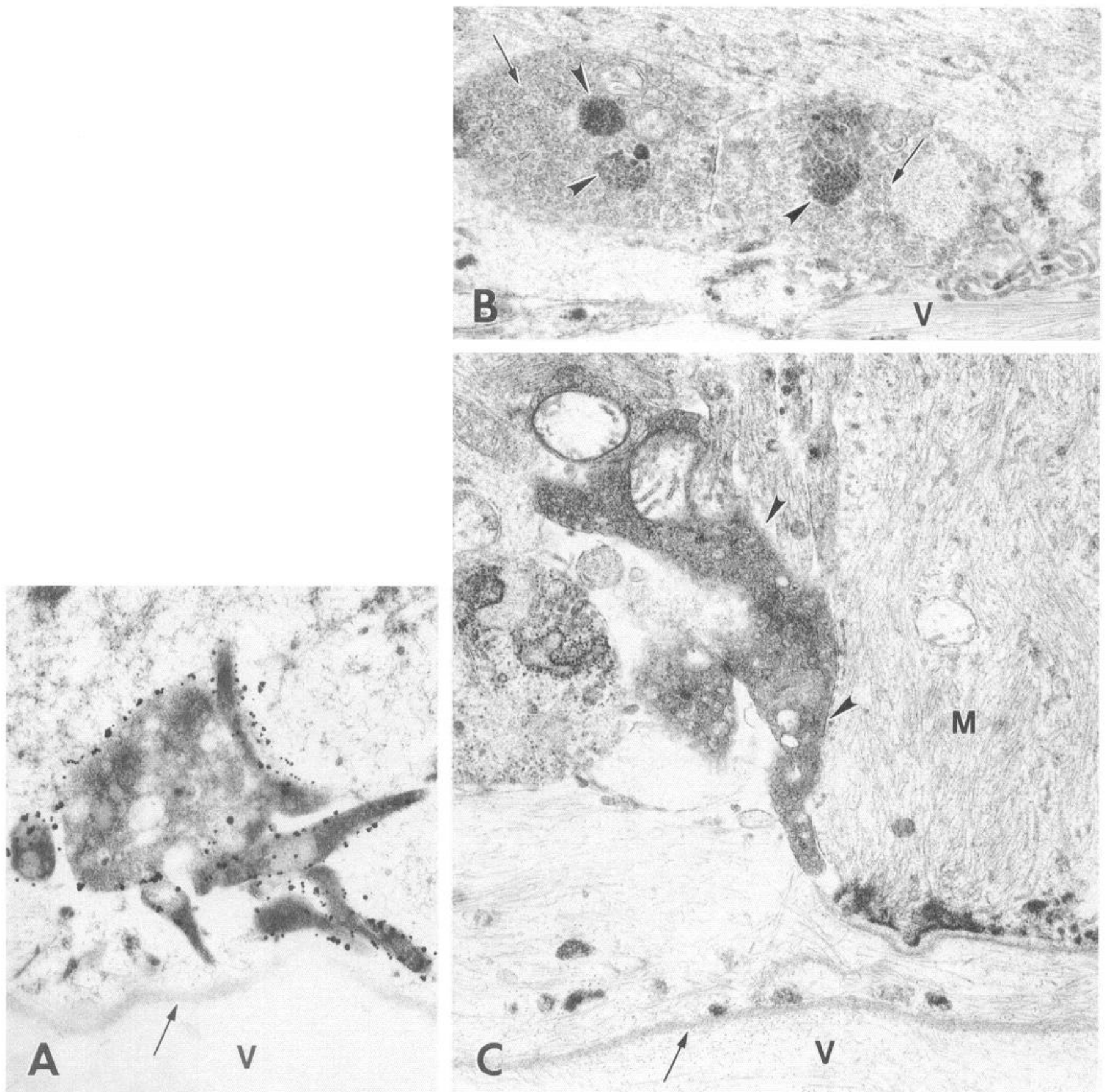

Figure 4. Electron micrographs of rod neurites in RP retinas. A, Immunogold labeling demonstrates rhodopsin in the surface membranes of rod neurites near the inner limiting membrane (arrow). $V$, vitreous. $B$, The rod neurites contain $50 \mathrm{~nm}$ synaptic vesicles (arrows) and dense multivesicular bodies (arrowheads). $V$, vitreous. $C$, The rod neurites (arrowheads) are closely apposed to a Müller cell process $(M)$, which is filled with intermediate filaments. Arrow, inner limiting membrane; $V$, vitreous. Magnification, $23,250 \times$.

rods at 10 months lacked outer segments, but the surface membranes and cytoplasm of their somata were well labeled with anti-rhodopsin (Fig. 5) and anti-recoverin, respectively. There was no evidence of neurite sprouting by these cells.

\section{Discussion}

This appears to represent the first demonstration of rod neurite sprouting in vivo. The neurites are formed by human rods that have survived significant death of neighboring photoreceptors. In rodent models of RP, the surviving rods show enlargement of their spherules in the outer plexiform layer (Jansen and Sanyal, 1992; Sanyal, 1993) and delocalization of rhodopsin and phototransduction proteins to the rod somata and synapses (Jansen et al., 1987; Nir et al., 1989, 1990; Cantera et al., 1990; Roof et al., 1994; Sung et al., 1994), yet these rods have not been noted to form neurites. We looked for neurites on surviving rods in old $r d s$ mice, using the same immunolabeling methods used on the human retinas, but also found no evidence of neurite sprouting. It is possible that rod neurites are detected in the human RP retinas because the process of rod cell death occurs 


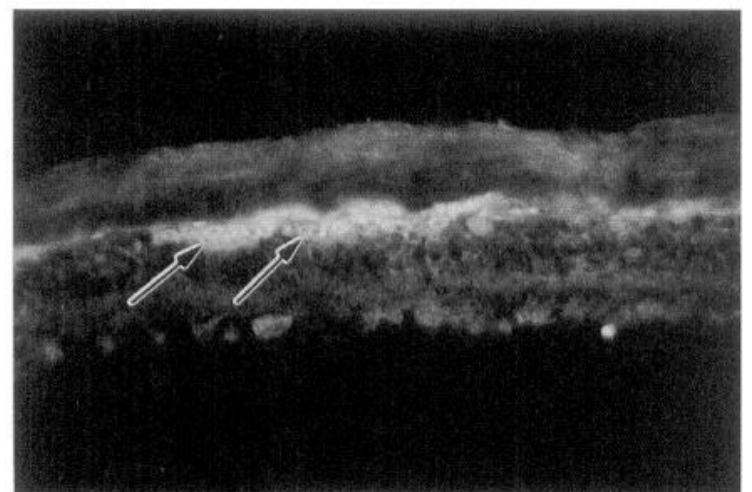

Figure 5. Retina of a 10-month-old rds mouse immunolabeled with anti-rhodopsin. The remaining rods lack outer segments, and their somata (arrows) show surface labeling for rhodopsin. No neurite sprouting is noted. Magnification, $230 \times$.

over years to decades rather than weeks to months, as found in rodents. Use of similar immunocytochemical methods on dystrophic retinas from 4-year-old dogs, the longest-lived animal models for human RP, also provided no evidence for neurite sprouting by the surviving rods (G. Aguirre, personal communication). Because rod neurite sprouting appears not to occur in nonhuman retinas, it may be due to properties peculiar to the human rods or to changes in the microenvironment unique to the RP retinas.

Neurite outgrowth is usually associated with the process of terminal differentiation of neurons and depends on interactions with neurotrophic factors, cell adhesion molecules, and the extracellular matrix. Mature neurons can respond to injury by sprouting neurites, possibly involving reactivation of a developmental mechanism, and their survival is promoted by various neurotrophic factors (Jelsma and Aguayo, 1994). Rod neurite sprouting in the RP retinas may result from survival factor upregulation in response to ongoing photoreceptor cell degeneration. In culture conditions, basic fibroblast growth factor (bFGF) promotes survival and differentiation of rods (Hicks and Courtois, 1992), and neurite outgrowth from other types of neurons (Hatten et al., 1988). Intravitreally injected bFGF rescues rat photoreceptors from light damage (Faktorovich et al., 1992), and the level of bFGF in retinal glia and photoreceptors is increased following optic nerve injury (Kostyk et al., 1994), consistent with the observation that optic nerve sectioning protects photoreceptors from light damage (Bush and Williams, 1991). Injected bFGF also delays photoreceptor degeneration in RCS rats (Faktorovich et al., 1990) and levels of photoreceptor bFGF are increased in degenerate $r d$ mouse retinas (Gao and Hollyfield, 1995). However, elevations in bFGF or in its receptor were not found in degenerate retinas of RCS rats (Connolly et al., 1992; Rakoczy et al., 1993), and the role of bFGF and other survival factors in the human retinal degenerations is at present unknown.

Rod axons in vivo are normally short and terminate in the outer plexiform layer. In culture, rods can form long, beaded neurites as found in the RP retinas (Araki et al., 1987; Kljavin and Reh, 1991; Gaur et al., 1992; Mandell et al., 1993; Hicks et al., 1994; Kljavin et al., 1994). Inhibitory factors that normally restrict rod axons to the outer plexiform layer may be missing in the culture conditions and in the diseased RP retinas. Alternatively, rod neurites may form in the RP retinas in response to changes in substrate factors, including cell adhesion molecules and components of the extracellular matrix (Bixby et al., 1994; Martini, 1994; Schachner, 1994). The rod neurites in the RP retinas were intimately associated with the surfaces of the hypertrophic Müller cell processes, and Müller glia are also the preferred substrate for neurite extension by mammalian rods in vitro (Kljavin and Reh, 1991; Gaur et al., 1992; Hicks and Courtois, 1992; Hicks et al., 1994). Following death of photoreceptors, Müller cells undergo reactive gliosis, including increased expression of GFAP (Eisenfeld et al., 1984; Milam and Jacobson, 1990; Li et al., 1994, 1995). The reactive Müller cells in the RP retinas have significantly increased surface membrane areas and associated molecules, including N-CAM (Z.-Y. Li, unpublished observations). Rods also show robust neurite outgrowth in culture on an N-CAM substrate (Kljavin et al., 1994). Thus, rod neurite formation in the RP retinas may be influenced by changes in the neighboring reactive Müller glia and their associated surface molecules.

In addition to rhodopsin, the rod neurites in the RP retinas contained several rod cytoplasmic proteins but not the outer segment membrane proteins, $r d s$ /peripherin, ROM-1, and the cGMP channel. Of the four proteins, rhodopsin is present in both the disk and plasma membranes of the outer segment, $r d s /$ peripherin and ROM-1 are found only in disk membranes, and the cGMP channel is restricted to the plasmalemma (Molday and Molday, 1993). The absence of $r d s$ /peripherin and ROM-1 in the neurites is consistent with the normal separation of rod disc membranes from the plasmalemma of the outer segment. The absence of delocalized cGMP channel in the surface membranes of the rod somata and neurites, if not due to masking, indicates that this outer segment plasmalemmal protein does not reach the neurites by simple backflow. It is possible that these rod proteins are sorted by different mechanisms in the inner segment, where separate post-Golgi vesicles are thought to carry rhodopsin to the outer segment and synaptophysin to the synapse region (Deretic and Papermaster, 1991; Holtzman, 1992; St. Jules et al., 1993). In the pathologic rods studied here, both rhodopsin and synaptophysin, as well as SV-2 synaptic vesicle protein, were routed into the neurites, suggesting that rhodopsin trafficking was abnormal and/or the mechanism that normally restricts rhodopsin to the outer segment had been lost (Spencer et al., 1988; Mandell et al., 1993).

Differential trafficking of rod outer segment membrane proteins is also found in experimentally detached cat retinas. These rods show delocalization of rhodopsin to the surface membranes of their inner segments, somata, and synapses (Lewis et al., 1991), while $r d s$ /peripherin is restricted to the truncated outer segments and to cytoplasmic vesicles in the inner segments (Fariss et al., 1993). The presence of rhodopsin in abnormal sites in the surface membrane does not lead to neurite formation in this model, however, and the axons of detached rods actually retract toward the somata (Erickson et al., 1983). It may be relevant that most photoreceptors die within several months after retinal detachment and by several weeks to months in the rodent models for RP, while the time scale for photoreceptor death in human RP spans a period of years to decades. The rod neurites may also persist for extended periods in the RP retinas, as they were present in retinas from donors ranging in age from 24 to 87 years (Table 1).

Because some loss of retinal ganglion cells occurs in RP (Stone et al., 1992), it seemed possible that the rod neurites had formed after transneuronal degeneration of cells that normally receive direct synaptic input from rod axons. Using specific im- 
munomarkers (Röhrenbeck et al., 1989; Grunert and Martin, 1991), we found that horizontal and rod bipolar cells were qualitatively normal, with no indication of transneuronal degeneration in regions of rod neurite sprouting, although some calbindin-positive horizontal cells had developed apical sprouts as found in the degenerate retinas of RCS rats (Chu et al., 1993). Double-labeling studies revealed that the rhodopsin-positive rod neurites extended through the calbindin-labeled band of horizontal cell processes and past the L-7-labeled rod bipolar cells to terminate at the inner limiting membrane. We also found no evidence by EM that the rod neurites formed specialized junctions with neurons, suggesting that the rod neurites lacked the ability to recognize normal signals for termination on horizontal and bipolar cell processes, or that these signals were missing from the postsynaptic cells.

Rods in the maculas of the RP retinas showed pathologic changes, including loss of their outer segments and rhodopsin delocalization to their somata. However, these rods did not form neurites, in spite of significant death of rods and cones in the macular regions. Cones throughout the RP retinas had truncated outer segments but they did not show opsin delocalization to their somata or neurite sprouting. In the peripheral parts of the RP retinas, some cone axons were abnormally elongated, reaching the inner plexiform layer. The cone axonal changes were much less extensive than the long, beaded neurites formed by the rods, but may similarly represent a response to growth/survival factors, as suggested for horizontal cell sprouts in RCS rat retinas (Chu et al., 1993).

Rhodopsin levels in RP retinas, as determined by fundus reflectometry, can be significantly higher than indicated by the reduced rod outer segment function (Perlman and Auerbach, 1981; Kemp et al., 1988). Thought originally to reflect loss of other phototransduction proteins in the rod outer segments, this observation may also reflect greatly increased amounts of rhodopsin present in the rod neurites in the inner retina. Transmission from rods and cones to inner retinal neurons can also be abnormal in RP, as assessed by electroretinographic and psychophysical techniques (Berson et al., 1968; Hood and Greenstein, 1990; Greenstein and Hood, 1992; Cideciyan and Jacobson, 1993; Falsini et al., 1994). It is not known if the rhodopsinpositive rod neurites and abnormal cone axons contribute to these functional abnormalities in RP.

Our findings have relevance for therapies aimed at restoring vision to RP patients by means of photoreceptor transplantation (Schuschereba and Silverman, 1992; Bok et al., 1993; Milam, 1993; Gouras et al., 1994). This approach is based on the assumption that transplanted normal photoreceptors can form synapses on surviving inner retinal neurons and reestablish a functional visual pathway. However, if changes in the retinal microenvironment lead to robust neurite sprouting by rods as shown here, the transplanted rods may not receive the signals for axon termination and synapse formation needed for their functional integration into the RP retinas. Perhaps the macular region should be considered for photoreceptor transplantation because rods and cones in this region do not appear to undergo axonal changes. Additional information is required on the pathologic alterations in RP retinas because at least one feature, rod neurite sprouting, does not appear to occur in the animal models.

\section{References}

Araki M, Iida Y, Taketani S, Watanabe K, Ohta T, Saito T (1987) Characterization of photoreceptor cell differentiation in the rat retinal cell culture. Dev Biol 124:239-247.
Berson EL, Gouras P, Gunkel RD (1968) Rod responses in retinitis pigmentosa, dominantly inherited. Arch Ophthalmol 80:58-67.

Bixby JL, Grunwald GB, Bookman RJ (1994) $\mathrm{Ca}^{2+}$ influx and neurite growth in response to purified $\mathrm{N}$-cadherin and laminin. $\mathrm{J}$ Cell Biol 127:1461-1475.

Bok D, Hageman GS, Steinberg RH (1993) Repair and replacement to restore sight. Report from the panel on photoreceptor/retinal pigment epithelium. Arch Ophthalmol 111:463-471.

Bush RA, Williams TP (1991) The effect of unilateral optic nerve section on retinal light damage in rats. Exp Eye Res 52:139-153.

Cantera R, von Schantz M, Chader GJ, Ehinger B, Sanyal S, van Veen $\mathrm{T}$ (1990) Postnatal development of photoreceptor-specific proteins in mice with hereditary retinal degeneration. Exp Biul 48:305-312.

Chu Y, Humphrey MF, Constable IJ (1993) Horizontal cells of the normal and dystrophic rat retina: a wholemount study using immunolabelling for the $28 \mathrm{kDa}$ calcium-binding protein. Exp Eye Res 57: 141-148.

Cideciyan AV, Jacobson SG (1993) Negative electroretinograms in retinitis pigmentosa. Invest Ophthalmol Vis Sci 34:3253-3263.

Connolly SE, Hjelmeland LM, LaVail MM (1992) Immunohistochemical localization of basic fibroblast growth factor in mature and de veloping retinas of normal and RCS rats. Curr Eye Res 11:10051017.

Deretic D, Papermaster DS (1991) Polarized sorting of rhodopsin on post-Golgi membranes in frog retinal photoreceptor cells. J Cell Biol 113:1281-1293.

Dryja TP (1992) Doyne lecture. Rhodopsin and autosomal dominant retinitis pigmentosa. Eye 6:1-10.

Eisenfeld AJ, Bunt-Milam AH, Sarthy PV (1984) Müller cell expression of glial fibrillary acidic protein after genetic and experimental photoreceptor degeneration in the rat retina. Invest Ophthalmol Vis Sci 25:1321-1328.

Erickson PA, Fisher SK, Anderson DH, Stern WH, Borgula GA (1983) Retinal detachment in the cat: the outer nuclear and outer plexiform layers. Invest Ophthalmol Vis Sci 24:927-942.

Faktorovich EG, Steinberg RH, Yasumura D, Matthes MT, LaVail MM (1990) Photoreceptor degeneration in inherited retinal dystrophy delayed by basic fibroblast growth factor. Nature 347:83-86.

Faktorovich EG, Steinberg RH, Yasumura D, Matthes MT, LaVail MM (1992) Basic fibroblast growth factor and local injury protect photoreceptors from light damage in the rat. J Neurosci 12:3554-3567.

Falsini B, Iarossi G, Porciatti V, Merendino E, Fadda A, Cermola S, Buzzonetti L (1994) Postreceptoral contribution to macular dysfunction in retinitis pigmentosa. Invest Ophthalmol Vis Sci 35:42824299.

Fariss RN, Matsumoto B, Fisher SK (1993) Confocal and EM immunolocalization of peripherin $/ r d s$ and opsin in degenerating photoreceptors. Invest Ophthalmol Vis Sci 34:768.

Gao H, Hollyfield JG (1995) Basic fibroblast growth factor in retinal development: differential levels of bFGF expression and content in normal and retinal degeneration $(r d)$ mutant mice. Dev Biol, in press.

Gaur VP, Liu Y, Turner JE (1992) RPE conditioned medium stimulates photoreceptor cell survival, neurite outgrowth and differentiation in vitro. Exp Eye Res 54:645-659.

Gouras P, Du J, Kjeldbye H, Yamamoto S, Zack DJ (1994) Long-term photoreceptor transplants in dystrophic and normal mouse retina. Invest Ophthalmol Vis Sci 35:3145-3153.

Greenstein VC, Hood DC (1992) The effects of light adaptation on L-cone sensitivity in retinal disease. Clin Vis Sci 7:1-7.

Grunert U, Martin PR (1991) Rod bipolar cells in the macaque monkey retina: immunoreactivity and connectivity. J Neurosci 11:2742-2758.

Hatten ME, Lynch M, Rydel RE, Sanchez J, Joseph-Silverstein J, Moscatelli D, Rifkin DB (1988) In vitro neurite extension by granule neurons is dependent upon astroglial-derived fibroblast growth factor. Dev Biol 125:280-289.

Hicks D, Courtois Y (1992) Fibroblast growth factor stimulates photoreceptor differentiation in vitro. J Neurosci 12:2022-2033.

Hicks D, Forster V, Dreyfus H, Sahel J (1994) Survival and regeneration of adult human photoreceptors in vitro. Brain Res 643:302305.

Hogan MJ, Alvarado JA, Weddell JE (1971) Histology of the human eye. Philadelphia: Saunders.

Holtzman E (1992) Membrane trafficking in neurons. Curr Opin Neurobiol 2:607-612. 
Hood DC, Greenstein V (1990) Models of the normal and abnormal rod system. Vision Res 30:51-68.

Humphries P, Farrar GJ, Kenna P (1993) Autosomal dominant retinitis pigmentosa: molecular, genetic and clinical aspects. In: Progress in retinal research, Vol 12 (Usborne NN, Chader GJ, eds), pp 231-245. Oxford: Pergamon.

Jansen HG, Sanyal S (1992) Synaptic plasticity in the rod terminals after partial photoreceptor cell loss in the heterozygous $r d s$ mutant mouse. J Comp Neurol 316:117-125.

Jansen HG, Sanyal S, DeGrip WJ, Schalken JJ (1987) Development and degeneration of retina in $r d s$ mutant mice: ultraimmunohistochemical localization of opsin. Exp Eye Res 44:347-361.

Jelsma TN, Aguayo AJ (1994) Trophic factors. Curr Opin Neurobiol 4:717-725.

Kemp CM, Jacobson SG, Faulkner DJ (1988) Two types of visual dysfunction in autosomal dominant retinitis pigmentosa. Invest Ophthalmol Vis Sci 29:1235-1241

Kljavin IJ, Reh TA (1991) Müller cells are a preferred substrate for in vitro neurite extension by rod photoreceptor cells. J Neurosci 11: 2985-2994.

Kljavin IJ, Lagenaur C, Bixby JL, Reh TA (1994) Ccll adhcsion molecules regulating neurite growth from amacrine and rod photoreceptor cells. J Neurosci 14:5035-5049.

Kostyk SK, D'Amore PA, Herman IM, Wagner JA (1994) Optic nerve injury alters basic fibroblast growth factor localization in the retina and optic tract. J Neurosci 14:1441-1449.

Lewis GP, Erickson PA, Anderson DH, Fisher SK (1991) Opsin distribution and protein incorporation in photoreceptors after experimental retinal detachment. Exp Eye Res 53:629-640.

Li Z-Y, Jacobson SG, Milam AH (1994) Autosomal dominant retinitis pigmentosa caused by the threonine-17-methionine rhodopsin mutation: retinal histopathology and immunocytochemistry. Exp Eye Res 58:397-408.

Li Z-Y, Possin DE, Milam AH (1995) Histopathology of bone spicule pigmentation in retinitis pigmentosa. Ophthalmology, in press.

Mandell JW, MacLeish PR, Townes-Anderson E (1993) Process outgrowth and synaptic varicosity formation by adult photoreceptors in vitro. J Neurosci 13:3533-3548.

Martini R (1994) Expression and functional roles of neural cell surface molecules and extracellular matrix components during development and regeneration of peripheral nerves. J Neurocytol 23:1-28.

Milam AH (1993) Strategies for rescue of retinal photoreceptor cells. Curr Opin Neurobiol 3:797-804.

Milam AH, Jacobson SG (1990) Photoreceptor rosettes with blue cone opsin immunoreactivity in retinitis pigmentosa. Ophthalmology 97: $1620-1631$.

Molday RS, Molday LL (1993) Isolation and characterization of rod outer segment disk and plasma membranes. In: Methods in neurosciences, Vol 15, Photorcceptor cells (Hargrave PA, ed), pp 131-150. San Diego: Academic.

Nir I, Agarwal N, Sagie G, Papermaster DS (1989) Opsin distribution and synthesis in degenerating photoreceptors of rd mutant mice. Exp Eye Res 49:403-421.

Nir I, Agarwal N, Papermaster DS (1990) Opsin gene expression during early and late phases of retinal degeneration in $r d s$ mice. Exp Eye Res 51:257-267.

Perlman I, Auerbach E (1981) The relationship between visual sensitivity and rhodopsin density in retinitis pigmentosa. Invest Ophthalmol Vis Sci 20:758-765.

Rakoczy PE, Humphrey MF, Cavaney DM, Chu Y, Constable IJ (1993) Express of basic fibroblast growth factor and its receptor in the retina of Royal College of Surgeons rats. A comparative study. Invest Ophthalmol Vis Sci 34:1845-1852.

Röhrenbeck J, Wässle H, Boycott BB (1989) Horizontal cells in the monkey retina: immunocytochemical staining with antibodies against calcium binding proteins. Eur J Neurosci 1:407-420.

Roof DJ, Adamian M, Hayes A (1994) Rhodopsin accumulation at abnormal sites in retinas of mice with a human $\mathrm{P} 23 \mathrm{H}$ rhodopsin transgene. Invest Ophthalmol Vis Sci 35:4049-4062.

Sanyal S (1993) Synaptic growth in the rod terminals after partial photoreceptor loss. In: Progress in retinal research, Vol 12 (Osborne NN, Chader GJ, eds), pp 247-270. Oxford: Pergamon.

Schachner M (1994) Neural recognition molecules in disease and regeneration. Curr Opin Neurobiol 4:726-734.

Schuschereba ST, Silverman MS (1992) Retinal cell and photoreceptor transplantation between adult New Zealand red rabbit retinas. Exp Neurol 115:95-99.

Spencer M, Detwiler PB, Bunt-Milam AII (1988) Distribution of membrane proteins in mechanically dissociated retinal rods. Invest Ophthalmol Vis Sci 29:1012-1020.

St. Jules R, Santa Hernandez M, Ungar F, Holcznan E (1993) Features of the polarity of the Golgi apparatus of frog photoreceptors: studies with lectins and with brefeldin A. Exp Eye Res 56:207-218.

Stone JL, Barlow WE, Humayun MS, de Juan E Jr, Milam AH (1992) Morphometric analysis of macular photoreceptors and ganglion cells in retinas with retinitis pigmentosa. Arch Ophthalmol 110:16341639.

Sung C-H, Makino C, Baylor D, Nathans J (1994) A rhodopsin gene mutation responsible for autosomal dominant retinitis pigmentosa results in a protein that is defective in localization to the photoreceptor outer segment. J Neurosci 14:5818-5833. 